\title{
The role of azathioprine in the management of retinal vasculitis
}

\begin{abstract}
Purpose Azathioprine is commonly used as a second-line immunosuppressive agent in the management of patients with retinal vasculitis. The aim of this study was to determine the efficacy of azathioprine by evaluating its effect on relapse rate, clinical outcome and maintenance steroid dose. Methods A retrospective analysis was performed of 34 patients presenting over 15 years to the Medical Eye Unit at St Thomas' Hospital. Relapse rate, steroid dosage, inflammatory score and visual acuity were assessed before and during treatment with azathioprine.

Results Thirty-four patients (23 male) aged 17-70 years (median 41.4, median 38 ) were enrolled. Fifteen had idiopathic retinal vasculitis, 12 had Behçet's disease, 2 sarcoidosis, 2 sympathetic ophthalmia, 1 birdshot retinochoriodopathy, 1 Still's disease and 1 Harada's disease. The principal indications for azathioprine treatment were uncontrolled disease despite appropriate steroid therapy in 28 patients $(82 \%)$ or to permit a decrease in steroid dosage in 6 patients $(18 \%)$. Frequency of relapse was assessed in 10 patients whose pre-treatment and treatment periods with azathioprine were of 1 year or greater. All were found to have a decreased replapse rate. In $60 \%$, reduction of steroid dosage was achieved. Ocular inflammatory score decreased in $56 \%$ of eyes and visual acuity was improved or maintained in $64 \%$. In 8 patients treatment had to be stopped due to side effects.

Conclusion Within the limitations of a retrospective study, we found azathioprine when used in combination with systemic steroids to be most effective at reducing the relapse rate of retinal vasculitis, but only partially effective in allowing a reduction in steroid dosage.
\end{abstract}

Key words Azathioprine, Immune suppression, Retinal vasculitis

In retinal vasculitis, systemic steroids are usually required for first-line immune suppression. Second-line immunosuppressive
A.J. GREENWOOD, M.R. STANFORD, E.M. GRAHAM agents such as cyclosporin A and azathioprine are used when systemic steroids are found to give an inadequate response, or when their use has resulted in unpleasant or hazardous side effects (hypertension, cataract, diabetes, centripetal obesity, skin changes, psychosis, osteoporosis, proximal myopathy, etc.). In our Unit, cyclosporin A is used when inflammation remains very active despite a dose of at least 25 $\mathrm{mg}$ prednisolone daily, whereas azathioprine is used when relapse of disease occurs with doses of prednisolone of $20 \mathrm{mg}$ daily. Before proceeding to second-line immune suppression, adequate doses of corticosteroid must be administered. Our current protocol is a highdose induction course lasting 9 weeks comprising $80 \mathrm{mg}$ oral prednisolone o.d. for 4 days, $60 \mathrm{mg}$ for 4 days followed by $40 \mathrm{mg}$ o.d. for 1 month and then $30 \mathrm{mg}$ o.d. for 1 month before reducing according to activity. The efficacy of this regime has been evaluated and found to offer optimal first-line treatment. ${ }^{1}$

Azathioprine (Imuran, GlaxoWellcome) has been used in the treatment of uveitis for at least 30 years. Early reports described the use of this drug in both isolated retinal vasculitis ${ }^{2}$ and Behçet's disease. ${ }^{3}$ Since then there have been several retrospective studies supporting its use in these and other ocular diseases, but the available data on the role of azathioprine remain scanty, although it is frequently used. Azathioprine has been reported to be an effective second-line immunosuppressive agent when used in combination with systemic prednisolone for uveitis. In one study, all patients treated with azathioprine exhibited a therapeutic response. ${ }^{4}$ This study also examined the efficacy of chlorambucil in combination with prednisolone and the number of patients in the group treated with azathioprine was therefore low. Azathioprine has been found to be the least toxic of the immunosuppressive drugs utilised to treat retinal vasculitis and has been used in the management of chronic disease, after control has been achieved with systemic steroids and cyclosporin. ${ }^{5}$ Improvements in disease control have also been described when azathioprine is used in conjunction with cyclosporin for posterior uveitis. ${ }^{6}$ However, cyclosporin has previously been associated with
A.J. Greenwood

M.R. Stanford

E.M. Graham

Medical Eye Unit

St Thomas' Hospital London, UK

Dr E.M. Graham Medical Eye Unit St Thomas' Hospital Lambeth Palace Road London SE1 7EH, UK

Tel: +44 (0)1719289292

Fax: +44 (0)1719228165

Supported by the IRIS Fund for the Prevention of Blindness 
a significant incidence of renal dysfunction, although at doses higher than those in common use today. ${ }^{7}$

Yazici et al. ${ }^{8}$ reported the results of a prospective controlled trial of azathioprine in Behçet's disease in 1990. Azathioprine therapy was found to be associated with a significantly reduced rate of progression of ocular complications, being superior to placebo in preserving visual acuity. A decreased rate of development of new eye disease in patients with Behçet's disease was also observed. The effect of azathioprine on the long-term prognosis of these patients has also recently been evaluated following further follow-up. ${ }^{9}$ Information was available for $85 \%$ of the patients originally enrolled and confirmed more frequent progression of eye disease and extraocular manifestations in the placebo group than in the azathioprine group. The frequency of blindness was $40 \%$ in the patients originally receiving placebo compared with $13 \%$ in those who had received azathioprine. Azathioprine (and cyclosporin) in combination with steroids for sympathetic ophthalmia has been found to control intraocular inflammation with lower steroid doses. ${ }^{10}$ Other authors have reported a $71 \%$ remission rate of patients with Behçet's disease treated with azathioprine. ${ }^{11}$ The toxicity of azathioprine has been extensively investigated. Of particular interest in a minority of patients with lymphopenia has been the effect of an inherited low level of the enzyme thiopurine methyltransferase (TPMT) resulting in high levels of azathioprine cytotoxic metabolites leading to profound myelosuppression. ${ }^{12}$ However, this enzyme deficiency occurs at a frequency of only 1 in $300 .^{13}$

In the literature concentrating on azathioprine, the patient population has usually been confined to those with Behçet's disease. ${ }^{8,9}$ In this study, we have carried out a retrospective analysis to examine the efficacy of azathioprine in retinal vasculitis with a variety of aetiologies including Behçet's disease, drawing on data from its use over many years in our Unit. In addition to therapeutic efficacy, the incidence and severity of side effects have also been examined.

\section{Patients and methods}

The records of patients with retinal vasculitis attending the Medical Eye Unit at St Thomas' Hospital, London, in the past 15 years were examined. Patients were included in the study who had a diagnosis of retinal vasculitis and had been treated with azathioprine. Both isolated cases and patients whose intraocular inflammation formed part of a recognised clinical entity were included. The diagnosis was made on the basis of characteristic clinical signs on ophthalmological examination, as well as the results of fluorescein angiography. Patients with infective or neoplastic causes were excluded.

All patients completed a course of high-dose systemic steroids according to our protocol and, following this, second-line immunosuppression with azathioprine was instituted. The principal indications for azathioprine treatment were: (1) an adequate response to steroid induction dose was followed by a relapse of disease when the prednisolone dose was reduced to below $20 \mathrm{mg}$; (2) steroid side effects developed that were either hazardous to or not tolerated by the patient.

Prior to commencement of azathioprine treatment, all patients were investigated with a full blood count, urea and electrolytes and liver function tests. The azathioprine regime comprised $50 \mathrm{mg}$ o.d. for 1 week, followed by $50 \mathrm{mg}$ b.d. for 1 week, increasing to $50 \mathrm{mg}$ t.d.s. continuous therapy in the absence of side effects. The maximal dose was adjusted to the nearest $50 \mathrm{mg}$ tablet to equal a daily dose of $2 \mathrm{mg} / \mathrm{kg}$ body weight. Patients were used as their own controls in analysis, comparisons being made between the pre-azathioprine treatment period, the period of azathioprine treatment, or the disease status at cessation of therapy if treatment had been discontinued. Relapse rates (number per year) before azathioprine treatment were assessed and compared with the period during which azathioprine had been added. A relapse was defined as an increase in vitreous cellularity as assessed by slit lamp biomicroscopy, or the appearance of fresh fundal lesions, and was often associated with an increase in visual symptoms (floaters or blurred vision). Although relapse was often accompanied by decreased visual acuity, this was not a prerequisite for its definition. Patients in whom lens opacity precluded a good view of the vitreous were excluded.

The average daily steroid dose after the 9 week highdose induction course during the pre-azathioprine treatment period was determined. This was then compared with the period after azathioprine had been introduced. Outcome at the end of the azathioprine treatment period was determined by measuring the overall change in inflammatory score and Snellen visual acuity, changes of 2 lines in Snellen acuity being regarded as significant. Inflammatory score was calculated using a weighted visual morbidity scale according to previously published criteria, each eye being assessed independently ${ }^{14}$ (Table 1). The incidence of side effects was examined; a history of skin rashes, gastrointestinal upset, warts and viral infections was specifically sought at each visit. Liver function tests and full blood counts were monitored every 6 weeks during azathioprine treatment.

Table 1. Ophthalmological criteria for grading activity of retinal vasculitis

\begin{tabular}{lcc}
\hline Ophthalmological feature & $\begin{array}{c}\text { Assessment } \\
\text { of severity }\end{array}$ & $\begin{array}{c}\text { Points } \\
\text { allotted }\end{array}$ \\
\hline Cells in vitreous & + & 1 \\
& ++ & 2 \\
Vascular sheathing & +++ & 3 \\
Macular oedema & + & 2 \\
& ++ & 5 \\
Vascular occlusion & + & 3 \\
& ++ & 7 \\
Neovascularisation & + & 5 \\
& ++ & 10 \\
& Present & 10 \\
\hline
\end{tabular}




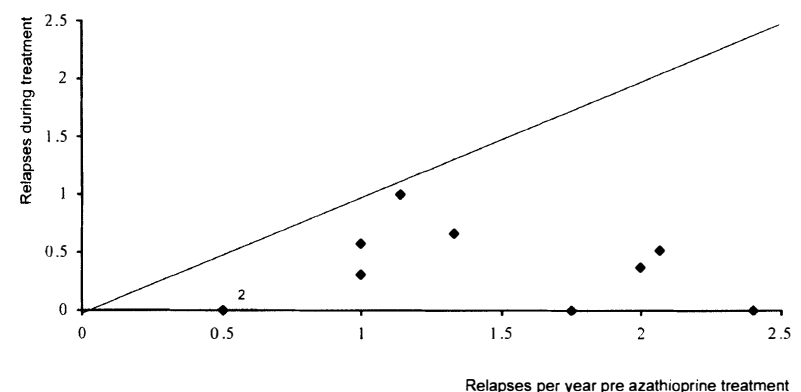

Fig. 1. Relapses per year before and during azathioprine treatment.

\section{Results}

\section{Patient characteristics}

The records of 34 patients who met the inclusion criteria were evaluated. Fifteen had idiopathic retinal vasculitis; of the remaining 19, 12 had Behçet's disease, 2 sarcoidosis, 2 sympathetic ophthalmia, 1 birdshot chorioretinopathy, 1 Still's disease and 1 Harada's disease. Twenty-three patients were male. Age range at inclusion was 17-70 years (mean 41.4, median 38). Treatment periods with azathioprine were variable, ranging from 1 week (treatment withdrawn due to nausea and vomiting) to 9 years (mean 25 months, median 18.5). The average pre-treatment period prior to the commencement of azathioprine was 30 months. In 28 patients azathioprine was introduced following uncontrolled disease on steroid maintenance and in 6 patients azathioprine was added in order to permit a decrease in steroid dose.

\section{Disease course and outcome}

\section{Relapse rate}

Analysis of relapse rate was confined to those patients who had been monitored in our Unit for a minimum of 1 year prior to the addition of azathioprine and whose treatment period with the drug had been 1 year or more at the time of study. Analysis was possible in 10 cases (comparing azathioprine treatment and pre-treatment periods). Seven of these patients had isolated retinal disease, 2 had Behçet's disease and 1 had birdshot chorioretinopathy. In 16 patients the pre-azathioprine treatment period was less than 1 year due to disease progression necessitating azathioprine therapy.

Azathioprine treatment periods of less than 1 year were found in 8 cases. This was the result of the development of side effects in 3 cases, the drug being judged to be ineffective in 1 case and disease quiescence being achieved in 1 further case. In 3 patients azathioprine was continuing at the time of the study, having been prescribed less than 1 year previously.

Relapse rates were found to decrease in all 10 patients during the treatment period when compared with their own pre-treatment rate of relapse (Fig. 1). In addition 4 patients managed to reduce their steroid dose; in 2 patients the disease came under control without any increase in steroids, but in 4 patients the steroid dose had

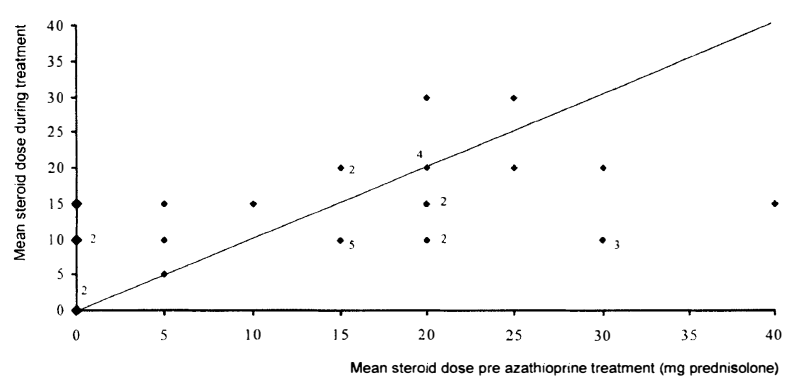

Fig. 2. Mean steroid dose before and during azathioprine treatment.

to be increased despite the introduction of azathioprine, which may have contributed to the improved disease control.

\section{Steroid dose}

Sufficient data were available to determine the mean steroid dose before and during azathioprine treatment in 32 patients (Fig. 2). The remaining 2 patients required azathioprine too early in their management to permit an accurate assessment of mean steroid dose in the preazathioprine treatment period. Decreased doses of prednisolone were achieved in 15 patients $(47 \%)$; doses remained unchanged in 7 patients $(22 \%)$ and increased in 10 patients ( $31 \%)$. In the 10 patients (19 eyes, 1 prosthesis) who required increased steroid doses, 12 eyes maintained or improved visual acuity. In 10 eyes (53\%) the inflammatory score was decreased and in 7 eyes $(37 \%)$ the inflammatory score was unchanged at the end of the treatment period. Those 10 patients requiring increased steroid dose did not have a greater improvement in inflammatory score than the group overall (inflammatory score decreased in $56 \%$ of eyes and remained unchanged in 36\%).

Eleven patients of this group of 32 had Behçet's disease. When compared with the rest of the group a higher proportion of these patients ( $45 \%$ compared with $24 \%$ ) required increased steroid doses, as their disease progressed despite the introduction of azathioprine (Table 2). In 6 patients of the group as a whole, azathioprine was specifically added as a steroid sparing agent as opposed to disease control being inadequate. In 4 of this group a reduction in the steroid dose was achieved, but steroid doses could not be reduced in the remaining 2 patients. In 1 of these patients azathioprine was subsequently discontinued after 3 months as no decrease in inflammation had been seen. In the second, disease quiescence was achieved after 8 months of therapy. The two most common diseases in this group of 32 patients were isolated retinal vasculitis (14 patients)

Table 2. Changes in steroid dose after addition of azathioprine for Behcet's disease and other disease groups (total 32 patients)

\begin{tabular}{lcc}
\hline $\begin{array}{l}\text { Steroid dose after } \\
\text { azathioprine introduction }\end{array}$ & $\begin{array}{c}\text { Behcet's disease } \\
(n=11)\end{array}$ & $\begin{array}{c}\text { Others } \\
(n=21)\end{array}$ \\
\hline Increased & $5(45 \%)$ & $5(24 \%)$ \\
Unchanged & $3(27 \%)$ & $4(19 \%)$ \\
Decreased & $3(27 \%)$ & $12(57 \%)$ \\
\hline
\end{tabular}


and Behcet's disease (11 patients). In 7 patients (50\%) with isolated disease, a reduced steroid dose was achieved after commencement of azathioprine. In the group of patients with Behçet's disease this was possible in only 3 cases $(27 \%)$ and a further $3(27 \%)$ required an equivalent dose.

\section{Inflammatory score}

Ocular inflammatory scores for each eye were assessed before commencing azathioprine therapy and compared with that at the end of azathioprine therapy (or at the time of assessment if azathioprine was continuing).

Sufficient information for accurate retrospective assessment of inflammatory score was possible in 61 eyes of 33 patients; assessment was not possible in a total of 4 eyes ( 3 eyes had no perception of light acuity and there were insufficient clinical data to calculate an inflammatory score, media opacity was present in 1 eye and 1 patient had an ocular prosthesis). Missing data in 1 patient prevented accurate analysis of the inflammatory score. At the end of azathioprine therapy 34 eyes were found to have a decreased inflammatory score $(56 \%)$, in $22(36 \%)$ the score was unchanged and $5(8 \%)$ had an increased level of inflammation. The inflammatory score improved in at least one eye in 21 patients (64\%). In 13 patients $(62 \%)$ the steroid dose was either reduced (5 patients, $24 \%$ ) or unchanged (8 patients, $38 \%$ ).

\section{Visual acuity}

Snellen visual acuity was assessed before commencing azathioprine and at the end of the treatment period. Sixty-seven eyes (34 patients, 1 prosthesis) were examined and used as their own controls. Twenty-three eyes (34\%) had an improved acuity (of at least 2 Snellen lines) at the end of follow-up, 21 eyes (31\%) maintained the same acuity and 23 eyes (34\%) had decreased acuity. The decrease was due to the development of cystoid macular oedema in 9 eyes, lens opacity in 2, recurrent vitreous haemorrhage in 2 , organised vitreous debris in 2 , optic atrophy in 1 , vein occlusion in 1 , cellophane maculopathy in 1 and rubeosis in 1 eye. In the remaining 4 eyes, there was a decrease in Snellen visual acuity of only 1 line for which no cause related to the ocular inflammatory disease was identifiable.

\section{Side effects}

Azathioprine therapy was continuing at the time of assessment in 10 patients. Twenty-four patients had azathioprine withdrawn and in 8 this was due to the development of unacceptable side effects (Table 3). In 13 cases disease quiescence permitted withdrawal of azathioprine and in 3 cases it was thought to be ineffective and subsequently discontinued. In 2 of the latter cases treatment periods were of 3 and 4 months' duration respectively. In the final case treatment eventually became ineffective after 42 months.
Table 3. Side effects during azathioprine treatment (34 patients)

No.

\begin{tabular}{lcc} 
Side effects & $\begin{array}{c}\text { No. } \\
\text { affected }\end{array}$ & $\begin{array}{c}\text { No. } \\
\text { requiring cessation } \\
\text { of treatment }\end{array}$ \\
\hline $\begin{array}{l}\text { Lymphopenia } \\
\left.\text { (normal range } 1.2-3.5 \times 10^{9} / 1\right)\end{array}$ & 17 & 0 \\
Abnormal liver function tests & 5 & 3 \\
Backache, fever, malaise & 1 & 1 \\
Dizziness & 1 & 1 \\
Joint pain & 1 & 1 \\
Skin rash & 1 & 1 \\
Nausea and vomiting & 1 & 1 \\
\hline
\end{tabular}

\section{The effect of lymphopenia}

Lymphopenia was defined as a level below the normal range of the hospital laboratory $\left(1.2-3.5 \times 10^{9} / 1\right)$. Seventeen patients $(50 \%)$ developed lymphopenia during treatment with azathioprine. The average steroid dose during the azathioprine treatment period of the patients who developed lymphopenia was $12.9 \mathrm{mg}$ prednisolone per day compared with $14.4 \mathrm{mg}$ prednisolone per day for those who did not develop this side effect.

The effect of azathioprine treatment on eye disease in this group was examined and compared with those who maintained a normal blood count during treatment. Snellen visual acuity was maintained or improved in 18 eyes $(55 \%)$ in the lymphopenic group compared with 25 eyes $(74 \%)$ in the other patients. Mean steroid dose was decreased during azathioprine therapy in 10 patients $(63 \%)$ in the lymphopenic group (with sufficient data) compared with 5 patients $(31 \%)$ in the remaining group. Inflammatory score was decreased in 17 eyes (59\%) in the lymphopenic group compared with 17 eyes (53\%) in the other patients. In 10 patients accurate assessment of relapse rate was possible (with at least a 12 month pretreatment period and 12 months of azathioprine therapy). All these patients were found to have a decreased relapse rate and 7 of these $(70 \%)$ developed lymphopenia.

\section{Additional immunosuppressive agents}

Eight patients needed additional immune suppression during the azathioprine treatment period. In all cases this was due to inadequate disease control on combined steroids and azathioprine therapy. Cyclosporin was the most commonly used additional drug and was prescribed in 5 cases ( 3 with Behçet's disease, 2 with isolated disease). In 2 patients (both with Behçet's disease) colchicine was used in addition to azathioprine.

\section{Discussion}

The major findings of this study were that all patients with appropriate follow-up achieved a decreased rate of relapse following the introduction of azathioprine. In addition, the majority of patients $(69 \%)$ did not require an increased steroid dose for disease control. Sixty-four per cent of patients had a decreased inflammatory score in at least one eye and the inflammatory score decreased 
in $56 \%$ of eyes. Visual acuity was maintained or improved in approximately two-thirds of eyes (65\%). We were not able to demonstrate that the development of lymphopenia is a prerequisite for response to azathioprine therapy. A greater proportion of the lymphopenic group (63\%) benefited from a decreased mean steroid dose during azathioprine therapy compared with the remaining patients $(31 \%)$. Lymphopenia was common (50\%) but it did not require the cessation of treatment in any patient. In the group of patients with adequate pre-treatment and treatment periods for accurate assessment of relapse rate, all were found to have a decreased rate of relapse on azathioprine, although 3 patients $(30 \%)$ had not developed lymphopenia. There were no life-threatening side effects. Abnormal liver function tests occurred in 5 patients $(15 \%)$ and treatment withdrawal was required in 3 of these cases (increased alanine transaminase, increased $\gamma$-glutaryl transferase and decreased albumin). All side effects resolved on withdrawal of azathioprine treatment.

Although there have been changes in the past decade with the development of more specific immunotherapy, the combination of azathioprine and steroids remains a reasonable first-line choice for the majority of patients with moderate intraocular inflammation and frequent relapse. We currently reserve cyclosporin for the patients with more aggressive disease. In this study we have presented the results of a retrospective descriptive analysis of our clinical experience of the management of retinal vasculitis over the past 15 years. Whilst the low incidence of this group of conditions has necessitated this type of analysis, it does have important limitations, which we recognise. The data presented are descriptive and difficult to analyse statistically, especially in view of the variety of clinical entities presented here. Whilst we have tried to minimise the effect of data being recorded by different observers, we accept that many doctors, both junior and senior, will have seen these patients during the period of study and in some cases it may have been difficult (even for highly experienced ophthalmologists) to differentiate between activity and damage. However, since there is relatively little published data on the use of azathioprine in this setting, we feel that this type of analysis does contribute valuable information.

Azathioprine has an accepted place as a second-line immunosuppressive agent for the treatment of retinal vasculitis. Prospective evidence exists to support its use in uveitis associated with Behçet's disease ${ }^{8}$ but not in other disease groups in which it is currently employed. To evaluate this treatment effectively in other ocular inflammatory conditions, randomised double-masked prospective analysis would be required. This is difficult for two reasons. Firstly, most specialists in this field accept that azathioprine is an invaluable agent in a group of conditions for which relatively few effective treatments are available. To withhold such a drug might therefore present ethical problems and indeed in the study of Behçet's disease ${ }^{8}$ many patients were withdrawn from the trial following progression of disease on placebo. Secondly, these conditions remain relatively rare and the recruitment of sufficiently large treatment and placebo control groups would be difficult to achieve.

We have examined the role of azathioprine in a group of patients with isolated retinal vasculitis, but also in patients with a variety of associated systemic diseases. Azathioprine was prescribed in these patients either to permit a decrease in the dose of systemic steroids or to improve disease control measured by rate of relapse and ocular inflammatory score. We have used doses of $50 \mathrm{mg}$ o.d., increasing to $50 \mathrm{mg}$ b.d. after 1 week, followed by $50 \mathrm{mg}$ t.d.s. after a further week, adjusting this maximal dose to the nearest $50 \mathrm{mg}$ tablet to equal a daily dose of $2 \mathrm{mg} / \mathrm{kg}$ of body weight. In our experience, this incremental introduction decreases the risk of gastrointestinal side effects and consequently increases compliance. We found azathioprine to be particularly effective in reducing the frequency of disease relapse. It was also effective in permitting a reduced steroid dose in approximately half of the patients. However, in patients with Behçet's disease this was more difficult to achieve and in this group approximately one-quarter (27\%) benefited from a decreased steroid dose. Yazici et al. ${ }^{8}$ showed azathioprine to be particularly effective in reducing relapse rate in patients with Behçet's disease, but we were unable to confirm this as only 2 of the patients in whom accurate relapse rates could be calculated had Behçet's disease.

In conclusion, this study supports the use of azathioprine as a safe and effective second-line immunosuppressive agent in the management of retinal vasculitis for both isolated disease and the other conditions described. We did not encounter any hazardous side effects that did not resolve on treatment withdrawal. Azathioprine seems particularly effective in reducing the rate of disease relapse, although it seems to have less efficacy when used as a steroid sparing agent.

\section{References}

1. Howe LJ, Stanford MR, Edelsten C, Graham EM. The efficacy of systemic corticosteroids in sight-threatening retinal vasculitis. Eye 1994;8:443-7.

2. Newell FW, Krill AE. Treatment of uveitis with azathioprine (Imuran). Trans Ophthalmol Soc UK 1967;87:499-511.

3. Lessof MH, Jeffrys DB, Lehner T, Sanders MD. Corticosteroids and azathioprine: their use in Behçet's syndrome. In: Lehner T, Barnes CG, editors. Behçet's syndrome: clinical and immunological features. London: Academic Press, 1979:218-26.

4. Andrasch RH, Pirofsky B, Burns RP. Immunosuppressive therapy for severe chronic uveitis. Arch Ophthalmol 1978;96:247-51.

5. Lightman S. Use of steroids and immunosuppressive drugs in the management of posterior uveitis. Eye 1991;5:294-8.

6. Vitale AT, Rodriguez A, Foster CS. Low dose cyclosporin A therapy in treating chronic, noninfectious uveitis. Ophthalmology 1996;103:365-73; discussion 373-4.

7. Graham EM, Sanders MD, James DG, Hamblin A, Kasp Grochowska E, Dumonde D. Cyclosporin in the treatment of posterior uveitis. Trans Ophthalmol Soc UK 1985;104:146-51. 
8. Yazici H, Pazarli H, Barnes CG, Tuzun Y, Silman A, Serdaroglu S, et al. A controlled trial of azathioprine in Behçet's syndrome. N Engl J Med 1990;322:281-5.

9. Hamuryudan V, Ozyazgan Y, Hizli N, Mat C, Yurdakul S, Tuzun Y, et al. Azathioprine in Behçet's syndrome: effects of long-term prognosis. Arthritis Rheum 1997;40:769-74.

10. Hakin KN, Pearson RV, Lightman SL. Sympathetic ophthalmia: visual results with modern immunosuppressive therapy. Eye 1992;6:453-5.

11. Kotter I, Durk H, Saal J, Fierlbeck G, Pleyer U, Ziehut M. Therapy of Behçet's disease. Ger J Ophthalmol 1996;5:92-7.
12. Anstey A, Lennard L, Mayou SC, Kirby JD. Pancytopenia related to azathioprine: an enzyme deficiency caused by a common genetic polymorphism [review]. J R Soc Med 1992;85:752-6.

13. Weinshilboum RM, Sladek SL. Mercaptopurine pharmacogenetics: monogenic inheritance of erythrocyte thiopurine methyltransferase activity. Am J Hum Genet 1980;32:651-62.

14. Dumonde DC, Kasp-Grochowska E, Graham E, Sanders MD, Faure JP, de Kozak, van Tuyen V. Anti-retinal autoimmunity and circulating immune complexes in patients with retinal vasculitis. Lancet 1982;2:787-92 